\title{
EL PROGRAMA DE MEDICINA EN LA UNIVERSIDAD PERUANA CAYETANO HEREDIA
} \section{THE PROGRAM OF THE MEDICAL CAREER IN UNIVERSIDAD PERUANA CAYETANO HEREDIA}

\author{
Manuel Gutiérrez Sierra ${ }^{1, a}$, María Paola Lucía Llosa Isenrich ${ }^{1, b}$
}

Facultad de Medicina, Universidad Peruana Cayetano Heredia. Lima, Perú

a Profesor asociado de la UPCH, grado de maestro en Educación. Director de Pregrado de Medicina; b Profesora principal de la UPCH. Doctor en Medicina. Decana de la Facultad de Medicina.

Recibido: 01-07-14; Aprobado: 03-09-14

\section{RESUMEN}

Los autores relatan el desarrollo del programa de estudios de la carrera de medicina en la Universidad Peruana Cayetano Heredia, especialmente en los últimos 15 años, tiempo en que dirigieron el Comité de Currículo. Ellos describen los fundamentos que han guiado el currículo de la carrera desde sus inicios hasta nuestros días; cómo se diseñó y evolucionó el perfil de egreso, así como las principales influencias en su diseño; cómo han evolucionado los procesos de enseñanza, aprendizaje y evaluación del aprendizaje; cómo se ha dado importancia al proceso de selección de estudiantes, y cómo se ha establecido el concepto de crédito académico ligado al trabajo real del estudiante y no solo al tiempo de trabajo presencial. Finalmente, señalan cómo ha mejorado la opinión que docentes y, especialmente, estudiantes tienen del programa, y los aspectos que deben ser mejorados en el futuro.

Palabras clave: Educación médica; Estudiantes de medicina; Enseñanza (fuente DeCS BIREME).

\begin{abstract}
The authors describe the development of the undergraduate medical curriculum at Universidad Peruana Cayetano Heredia, especially over the last 15 years, when they led the Curriculum Committee. They describe the principles that have guided the curriculum development process up to this day; how the competency outcomes were designed and evolved as well as the main influences on the process; how the teaching, learning and assessment process has evolved; how the student selection and admission has been valued; and how the concept of credit has been linked to the work that students need to learn. Finally, they comment on how faculty and students appreciate the program and on the improvement required in the future.
\end{abstract}

Key words: Education, medical; Students, medical; Teaching (source: MeSH, NLM).

\section{INTRODUCCIÓN}

La Universidad Peruana Cayetano Heredia fue fundada el 22 de septiembre de 1961, como Universidad Peruana de Ciencias Médicas y Biológicas, e inició actividades académicas de pregrado de Medicina en mayo del año 1962. En su historia ha marcado diferencias con otros currículos, por ejemplo, al implementar el externado (1970) y luego el internado rural (1971). El externado es un año de práctica previo al internado donde se integran los equipos asistenciales en las sedes docentes, esto es imitado, en alguna medida, por otras escuelas de Medicina.

El currículo ha sido actualizado y mejorado por distintas comisiones creadas con tal propósito. En el año 2001 se formó la actual Comisión de Currículo con el propósito de iniciar un proceso de mejoramiento continuo del currículo de Medicina. En ese mismo año, la Facultad de Medicina realizó una encuesta de autoevaluación, la cual reveló que la mayoría de estudiantes consideraban que el programa académico era bueno, incluyendo a $7 \%$ que lo consideraban excelente, pero $20 \%$ lo consideraban malo. La mayoría de docentes y estudiantes reconocía que la Facultad promovía la adquisición de conocimientos y habilidades, así como actitud ética, pero no promovía suficiente interés por el autodesarrollo, la disposición de servicio, ni la cultura humanística.

La comisión hizo suya la percepción de que, en general, la formación en ciencias básicas era insuficiente, que se había avanzado poco en integración de contenidos y disciplinas, que la formación en valores dependía exclusivamente del currículo oculto y que se mantenía un modelo educativo centrado en la transferencia de información. 


\section{FUNDAMENTOS DEL CURRÍCULO DE MEDICINA}

En este artículo nos referimos al currículo como el conjunto de experiencias diseñadas con el propósito que la persona que las vive (estudiante) evolucione de un estado inicial (ingresante) a uno final (egresado). Esto implica la construcción de competencias para realizar las tareas propias de la profesión, que integran conocimientos, habilidades, valores y actitudes.

El currículo así entendido se hace explícito a través del plan curricular, sílabos y documentos de gestión, e incluye a los docentes, a los estudiantes, a las estrategias y tecnología educativas, a los ambientes en que ocurren las experiencias, a los equipos e insumos, $y$ a la organización que lo hace viable. El currículo incluye y trasciende al plan de estudios, siendo este último el listado de cursos y rotaciones presentados de una manera ordenada.

Los lineamientos básicos que guiaron el plan curricular aprobado en 2008 siguen vigentes y consideran los siguientes aspectos:

1. Promover el desarrollo de los estudiantes como seres humanos, cultivando su sensibilidad y solidaridad hacia los demás.

2. Lograr aprendizaje significativo, para lo cual el objeto de aprendizaje debe ser coherente y relevante para el estudiante, buscando el desarrollo de competencias. Este solo puede darse si la educación está centrada en el alumno, lo que implica un cambio sustancial en el rol del profesor.

3. Promover la comprensión y el manejo integral de los problemas de salud, en sus dimensiones biológica, ecológica, social, cultural, psicológica y espiritual.

4. Formar un médico competente para resolver y responder adecuadamente a la demanda de problemas de salud individuales y colectivos, haciendo énfasis en el manejo preventivopromocional, con la potencialidad de autoaprendizaje continuo y la posibilidad de especializarse en el futuro.

5. Promover el desarrollo de intereses y habilidades no académicas de los estudiantes.

6. Definir un sistema de evaluación que valore y promueva el desarrollo de competencias.

7. Promover el desarrollo de los docentes, asegurando que estén preparados para asumir su nuevo rol como motivadores y facilitadores del proceso.

El desarrollo o construcción de competencias es un proceso que implica la adquisición de conocimientos, la movilización de estos en situaciones de complejidad creciente y de similitud cada vez mayor con la situación de desempeño. La articulación entre el sistema de salud del país y la educación médica determina que los egresados de la carrera de Medicina tienen que graduarse con la experiencia clínica suficiente para mostrar un nivel de logro de la competencia médica que le permita ejercer sin supervisión. Esto está en marcado contraste con la realidad de países desarrollados, en los que los egresados deben cumplir programas de especialización de varios años antes de ejercer sin supervisión. Por ello, es imprescindible que el currículo asegure horas suficientes de práctica supervisada.

La psicología del aprendizaje y su aporte en el reconocimiento de la importancia de la experiencia como fenómeno que motiva e inicia el aprendizaje ha tenido gran influencia en el desarrollo de los últimos años, así como el aporte de la neurociencia, que en la última década ha hecho descubrimientos clave para entender la base biológica de la función mental, incluyendo la memoria.

\section{EL PERFIL DE EGRESO}

En el año 2002 incorporamos el concepto de perfil académico-profesional como el objetivo de la carrera, asumiendo, además, que el perfil está definido por las competencias que se espera que el estudiante construya en sus años de pregrado. En su primera versión, el perfil estuvo definido como de la siguiente manera

"Es objetivo del proceso educativo de la Facultad de Medicina Alberto Hurtado la formación de ciudadanos y profesionales médicos, cuyas sólidas bases humanista y científica le permitan:

Progresar en su construcción como ser humano: integro, responsable, sensible, reflexivo, crítico y ético, con salud física, mental y espiritual, con relaciones humanas saludables y solidarias, con capacidad para integrarse a la sociedad, analizarla y transformarla democráticamente.

Ser un médico general, con las competencias para:

Ser líder servidor, habiendo desarrollado una vocación de servicio y asumiendo la medicina no solamente como un medio de vida.

Considerar en el ejercicio profesional las variables biológicas, psicológicas, socioculturales y ecológicas de las personas que atiende y de los problemas de salud.

Prevenir, diagnosticar y tratar con enfoque integral los problemas de salud individual y colectiva prevalentes, con eficiencia, eficacia, calidad $y$ equidad, y con conciencia de sus capacidades y limitaciones. 
Ser educador, motivando y persuadiendo a las personas a asumir su responsabilidad en la solución de sus problemas de salud.

Administrar servicios de salud de baja complejidad.

Utilizar racionalmente los recursos.

Integrar equipos de trabajo multidisciplinarios e interprofesionales.

Emplear en su trabajo los adelantos de la informática y la tecnología.

Orientarse a la investigación, aportando conocimiento, soluciones y tecnología.

Actuar de acuerdo al Código de Ética y Deontología de la profesión.

Evaluarse permanentemente y continuar su desarrollo personal y profesional"

Poco después vimos que, si bien el perfil redactado reflejaba nuestras intenciones, no era suficientemente explícito ni facilitaba la evaluación de los logros de los estudiantes. En enero de 2003 hicimos una descripción de los que llamamos la "competencia final", sobre la base de la medicina centrada en el paciente ${ }^{(1)}$ y siguiendo los lineamientos del enfoque integral de los problemas de salud individual y colectiva. En esta competencia final describimos cinco dimensiones básicas de la competencia del médico: diagnostica, trata, promueve salud, aprende continuamente y demuestra conducta ética.

En el año 2004 tomamos contacto con el trabajo desarrollado por el grupo curricular de los decanos de las facultades de medicina escocesas, basado en resultados de aprendizaje (learning outcomes) ${ }^{(2,3)}$. Este modelo define los resultados de aprendizaje en tres círculos concéntricos, donde el círculo interno describe "lo que el médico es capaz de hacer", el intermedio representa "cómo el médico enfrenta las tareas del círculo interno" y el externo "representa el desarrollo de los atributos personales del individuo". El círculo interno tiene siete resultados: habilidades clínicas, procedimientos, investigación de los problemas del paciente, atención (management) del paciente, promoción de salud y prevención de enfermedad, comunicación, y habilidades para el manejo de información. El círculo intermedio tiene tres resultados: comprensión de los principios de las ciencias sociales, básicas y clínicas; actitudes apropiadas, comprensión de la ética y de las responsabilidades legales; $y$ habilidades de toma de decisiones, razonamiento y juicio clínico. El círculo externo tiene dos resultados: rol del médico en el sistema de salud, y desarrollo personal.

En abril del año 2005 realizamos la tarea de definir los elementos de competencia para cada uno de los 12 resultados siguiendo el modelo escocés y, en noviembre del mismo año, los incorporamos al plan curricular, entonces en construcción. También en el año 2005 recibimos la invitación de la Asamblea Nacional de Rectores para representar al Perú en el Proyecto Tuning - América Latina, por el área temática (carrera) de Medicina. El proyecto sigue los fines y la metodología de su similar europeo, que busca establecer un espacio común de educación superior.

El proyecto Tuning - América Latina tiene cuatro grandes líneas de trabajo: competencias (genéricas y específicas de las áreas temáticas); enfoques de enseñanza, aprendizaje y evaluación de estas competencias; créditos académicos; y calidad de los programas. Las competencias genéricas fueron definidas en las reuniones de Buenos Aires y Belo Horizonte, por las áreas temáticas entonces participantes, que aún no incluían a medicina. El área de medicina planteó sus competencias específicas en la reunión de Costa Rica, en febrero de 2006. Estas fueron consultadas a estudiantes, académicos, egresados y empleadores de todas las universidades participantes. Después de la consulta se acordó 63 competencias específicas para medicina, descritas en términos de capacidades (4). Estos resultados fueron mostrados en la reunión de Bruselas, de junio de 2006.

La Comisión procedió luego a comparar las competencias originalmente aprobadas con las acordadas por Tuning. El grado de coincidencia entre ambas fue muy grande. Después de haber completado la revisión hicimos una selección final, que fue sometida a consulta a un grupo más reducido de estudiantes, egresados y docentes. El resultado de este trabajo se muestra en el plan curricular aprobado en enero de 2008, como perfil académico profesional. En este perfil definimos la competencia del médico conformada por tres dimensiones:

- Lo que el médico hace: práctica clínica e investigación,

- Cómo realiza su práctica: sobre bases científicas y éticas, con actitud profesional y ubicado en el sistema de salud, y

- El médico como persona: demuestra comportamiento ético, aprende permanentemente, resuelve problemas, trabaja en equipo, asume liderazgo, contribuye al conocimiento y se desempeña en contextos diversos.

El resultado fue una formulación altamente integrada de la competencia del médico, que además facilitó la evaluación de desempeños relevantes también integrados, pero quedaron cabos sueltos en cuanto al aprendizaje y evaluación de algunos de los elementos que las componen y no se hizo explícitos los problemas que los egresados deberían ser capaces de atender 
Tabla 1. Competencias generales de la Universidad Peruana Cayetano Heredia y específicas de su escuela de medicina.

\begin{tabular}{|c|c|}
\hline $\begin{array}{l}\text { Competencias generales } \\
\text { (toda la universidad) }\end{array}$ & $\begin{array}{l}\text { Competencias específicas } \\
\text { (carrera de medicina) }\end{array}$ \\
\hline 1. Demuestra comportamiento y compromiso & 1. Realiza la práctica clínica: \\
\hline 2. Es crítico, innovador y creativo. & $\begin{array}{l}\text { a. Diagnostica y establece el pronóstico de los problemas de salud } \\
\text { individual y colectiva prevalentes, con enfoque integral, eficiencia, } \\
\text { eficacia, calidad y equidad, y con conciencia de sus capacidades y } \\
\text { limitaciones. }\end{array}$ \\
\hline $\begin{array}{l}\text { 3. Asume liderazgo, gestiona actividades y } \\
\text { proyectos trabajando en equipo. }\end{array}$ & $\begin{array}{l}\text { b. Indica y realiza el tratamiento de los problemas de salud individual y } \\
\text { colectiva prevalentes, con enfoque integral, con eficiencia, eficacia, } \\
\text { calidad y equidad, y con conciencia de sus capacidades y limitaciones. }\end{array}$ \\
\hline $\begin{array}{l}\text { 4. Se comunica eficaz y correctamente en } \\
\text { castellano y utiliza tecnologías de información } \\
\text { y comunicación. Se comunica en inglés en } \\
\text { situaciones y temáticas cotidianas. }\end{array}$ & $\begin{array}{l}\text { c. Realiza la promoción de salud y la prevención de enfermedades con } \\
\text { enfoque integral, con eficiencia, eficacia, calidad y equidad, y con } \\
\text { conciencia de sus capacidades y limitaciones. }\end{array}$ \\
\hline 5. Resuelve problemas con rigor científico. & $\begin{array}{l}\text { d. Aplica el razonamiento y juicio clínico basados en la evidencia y en } \\
\text { la experiencia. }\end{array}$ \\
\hline $\begin{array}{l}\text { 6. Asume su desarrollo personal y profesional y } \\
\text { se compromete con él. }\end{array}$ & e. Se desempeña en el sistema de salud. \\
\hline $\begin{array}{l}\text { 7. Demuestra compromiso y responsabilidad con } \\
\text { su entorno social y medioambiente. }\end{array}$ & f. Establece la relación médico-paciente. \\
\hline \multirow[t]{2}{*}{$\begin{array}{l}\text { 8. Se desempeña eficientemente en contextos } \\
\text { socio-culturales y profesionales diversos. }\end{array}$} & 2. Diseña, realiza y publica investigación. \\
\hline & 3. Administra y gestiona un establecimiento básico de atención. \\
\hline
\end{tabular}

y resolver. Por ello, el modelo fue revisado en 2009, incluyendo la relación de problemas de salud individual y colectiva. El resultado fue nuevamente sometido a consulta a estudiantes y docentes en diciembre de 2009. El producto de esta formulación y consulta es la base del perfil de egreso que forma parte de la actualización curricular curso. Este está formulado tomando como base las competencias genéricas y específicas que los estudiantes deben demostrar antes de graduarse (Tabla 1) y estas están compuestas por elementos evaluables.

\section{PROCESOS DE ENSEÑANZA- APRENDIZAJE Y EVALUACIÓN}

Durante años se siguió el modelo que primero se aprende (la teoría), luego se practica $y$, finalmente, se evalúa. Aún hay académicos y estudiantes que encuentran este modelo perfectamente lógico. Sin embargo, el estado actual del conocimiento nos muestra que el aprendizaje muchas veces empieza con la experiencia, no necesariamente con los conceptos teóricos; que la motivación es esencial, que los escenarios y situaciones de aprendizaje tienen un efecto crítico en lograr aprendizaje significativo, que la evaluación es inseparable del aprendizaje, que es el estudiante quien aprende y el profesor quien debe facilitar el aprendizaje.

El externado e internado reúnen condiciones para ser escenarios ideales de aprendizaje, gracias a la larga asociación formal de la Facultad con las sedes de sus campos clínicos, con los docentes que trabajan en ellos y a la existencia de programas de especialización en los mismos. Estos dos últimos años de la carrera son, además, el escenario apropiado para evaluar el desarrollo de competencias, lo que se hace desde el año 2005 mediante la observación del desempeño en servicio, con instrumentos (rúbricas) creados para tal fin. La interacción permanente con los estudiantes nos permitió reconocer que ellos mismos tienen más y mejores oportunidades de evaluar su desempeño y el de sus compañeros. Por ello, desde el año 2013 estamos aplicando interevaluación en el internado y externado, lo que está produciendo resultados altamente satisfactorios.

Cuarto y quinto son años de transición entre escenarios de aprendizaje controlados (aulas, laboratorios) y establecimientos de salud de diferente complejidad. La estructura de los cursos de esos años se caracteriza por el balance entre aprendizaje en los servicios asistenciales, en grupos pequeños, y el aprendizaje en aula. El aprendizaje en aula incluye clases expositivas y discusiones de casos clínicos seleccionados para tal fin. El aprendizaje en los servicios asistenciales combina la interacción con pacientes y con los equipos de trabajo asistenciales, así como presentaciones y discusiones en relación a los pacientes atendidos.

La responsabilidad de asegurar la integración entre el aprendizaje en la práctica y en el aula recae en los tutores. Los cursos de clínica médica, pediátrica, 
quirúrgica y ginecoobstétrica cuentan con actividades de tutoría semanales. El tutor es responsable de revisar las historias clínicas confeccionadas por los estudiantes, promover el desarrollo del juicio clínico entre sus estudiantes y asegurar que la experiencia de aprendizaje sea integral. Desde el año 2013 han incorporado la tarea de identificar estudiantes que puedan requerir apoyo personalizado y referirlos al área de asuntos estudiantiles para las gestiones correspondientes.

En el 2014 hemos inaugurado ambientes para realizar actividades de simulación de consulta o atención ambulatoria y para el aprendizaje de procedimientos con maquetas. Esto ha permitido gestionar eficaz y eficientemente actividades que venían realizándose de manera aislada en varios cursos. El funcionamiento de este centro ha permitido reemplazar exámenes prácticos, poco objetivos y reproducibles, con evaluaciones clínicas objetivas y estructuradas (ECOE) en el curso de Introducción a la Clínica. Hemos decidido, además, que los estudiantes realizarán el aprendizaje de procedimientos y este será certificado en simulación antes de empezar el externado.

En el año 2007 introdujimos seminarios de grupos pequeños en cursos integrados de tercer año (Estructura y Función, Infección e Inmunidad). En estos seminarios aplicamos el estudio de casos y redujimos la cantidad de clases expositivas a dos horas semanales. Esto significó un cambio radical en relación al modelo tradicional centrado en clases teóricas y prácticas de laboratorio. La retroalimentación recibida de los estudiantes mostró que muchos de ellos necesitaban más tiempo del estimado debido a lo empinado de la curva de aprendizaje. Por otro lado, las evaluaciones de conocimientos aplicadas al ingreso a cuarto año mostraron que el rendimiento no había disminuido, pero tampoco había mejorado en relación a antes de introducir los seminarios. Por ello, decidimos aumentar las clases inicialmente a cuatro y desde el 2013 a seis horas semanales, para facilitar a los estudiantes la selección y organización de conocimientos. Desde la introducción de esta modificación hemos podido comprobar una mejoría significativa en el rendimiento de los estudiantes en la evaluación mencionada, como se muestra en la Figura 1.

Otra innovación relevante fue el trabajo en la comunidad, aplicado desde el año 2007 en los cursos de Introducción a Ciencias Sociales y Antropología, y en centros de salud de primer nivel, en el curso de Salud Comunitaria. El aprendizaje en la comunidad nació bajo la premisa de que los estudiantes deben aprender de su experiencia al trabajar en la comunidad, con la comunidad y respetando la voluntad de la comunidad.

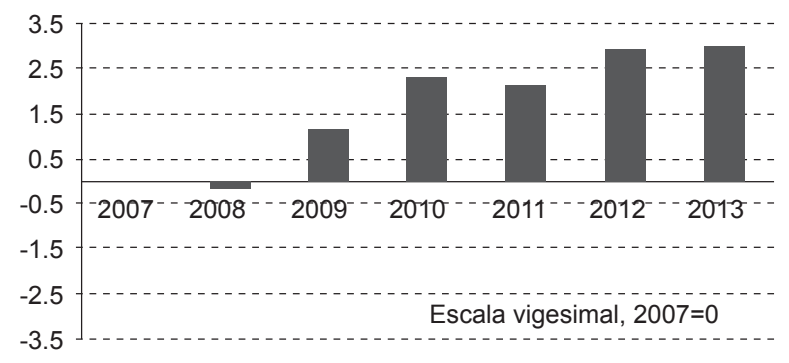

Figura 1. Evolución de la nota promedio sobre los contenidos de las ciencias preclínicas en el examen al inicio de cuarto año. La nota promedio del año 2007 se iguala a cero. Nota expresadas en puntos de escala vigesimal.

Este trabajo empezó en el distrito de Independencia y se trasladó a la comunidad de Manchay, gracias a la apertura de su párroco.

Aunque reconocemos la importancia del desarrollo de competencias y su evaluación, es importante asegurar la adquisición de conocimiento que permitirá a los graduados desempeñarse ante problemas diversos y en contextos diversos. Por ello, fuimos partícipes e impulsamos la creación y desarrollo del Examen Nacional de Medicina (ENAM). Sin embargo, debemos reconocer que, desde que el puntaje obtenido por los internos tiene un peso en determinar el orden de elección de plazas para el SERUMS, los internos tienen que prepararse para rendirlo, con lo que los resultados pierden validez como indicador del aprendizaje directamente relacionado con el programa académico ${ }^{(5)}$. Para suplir esta pérdida, nuestros internos rinden desde hace tres años el examen International Foundations of Medicine (IFOM). El examen es desarrollado por el National Board of Medical Examiners (NBME) de los Estados Unidos y, si bien tiene algunas limitaciones, como no incluir contenidos de medicina tropical, ha sido bien valorado por nuestros internos que lo han rendido, y estamos utilizando los resultados para mejorar los contenidos de cuarto y quinto años.

\section{PROCESOS DE SELECCIÓN}

A pesar que los procesos de selección de estudiantes no son responsabilidad directa de la Facultad de Medicina, los resultados de los mismos nos son de gran interés, porque de ellos depende contar con estudiantes potencialmente exitosos. El intercambio de información y opiniones entre la Dirección de Carrera de Medicina y la Oficina de Admisión, Matrícula y Registro Académico, ha ido modelando los procesos de selección de manera que todos los postulantes a Medicina serán sometidos a evaluación de conocimientos, de aptitud académica y 
a entrevista personal. La idea es que la evaluación de conocimientos sea aplicada con el propósito demostrar suficiencia, pero la nota que decida el ingreso dependa de las pruebas de aptitud académica y entrevista personal.

\section{EL CRÉDITO ACADÉMICO Y EL TIEMPO DE LOS ESTUDIANTES}

En la Facultad de Medicina se reconoció, el año 2009, la importancia del uso apropiado del tiempo para asegurar condiciones óptimas de aprendizaje, así como que las metodologías de enseñanza y aprendizaje son tan variadas que no es posible asumir un número constante de horas de estudio o trabajo independiente por cada hora de trabajo presencial. Por ello, se acordó que los créditos académicos deben ser un reflejo del trabajo de los estudiantes, considerando tanto las horas presenciales (programadas) como el estudio y trabajo independiente o en grupo. En ese año iniciamos en la Dirección de Pregrado el trabajo de hacer un seguimiento del uso del tiempo por los estudiantes. La recopilación de información es semanal y cada estudiante de los tres primeros años registra en una ficha las horas que dedica a estudiar en grupo o individualmente y a realizar tareas o trabajos. Esto nos ha permitido disponer de información confiable y oportuna para regular las actividades de aprendizaje, en coordinación con los responsables de los cursos. Es necesario mencionar que la mayoría de los estudiantes proporcionan la información solicitada.

\section{EL FUTURO}

En el año 2012 volvimos a aplicar la encuesta realizada en el año 2001, con la finalidad de recoger la opinión de estudiantes y docentes sobre el programa. Fue bueno averiguar que el $20 \%$ de estudiantes que había mostrado insatisfacción con el programa en el 2001 se había reducido a $6 \%$ y que quienes lo consideraron excelente aumentaron del 7 al $17 \%$. Otros aspectos que promueve el programa que fueron mejor evaluados por los estudiantes en 2012 fueron disposición para el servicio, interés por autodesarrollo y aprendizaje continuo, el desarrollo de habilidades, la adquisición de conocimientos y la conducta ética. Por lo general, los docentes compartieron opiniones con los estudiantes, con excepción de la conducta ética, acerca de la cual los docentes opinan que ha disminuido discretamente.

Estamos desarrollando líneas de trabajo para mejorar la formación en ética y profesionalismo de los estudiantes, así como para promover la iniciación en investigación desde los primeros años de carrera para los estudiantes que tengan la inclinación para ello. Finalmente, aún necesitamos mejorar la evaluación del aprendizaje, tanto del conocimiento como del desempeño, necesitamos alinear mejor los resultados esperados de aprendizaje con las experiencias de aprendizaje y la evaluación del mismo, y también necesitamos que los docentes estén más familiarizados con los principios y las prácticas actuales en materia de educación y que algunos de ellos vean la docencia como su principal responsabilidad. Mejorar estos aspectos del currículo debe ser nuestra prioridad en los próximos años.

Conflictos de interés: los autores declaran no tener conflictos de interés.

Fuentes de financiamiento: autofinanciado.

\section{REFERENCIAS BIBLIOGRÁFICAS}

1. Stewart M, Belle Brown J, Wayne Weston W, McWhinney IR, McWilliam CL, Freeman TR. PatientCentered Medicine: Transforming the Clinical Method. California: SAGE Publications; 1995.

2. Smith SR, Dollase R. AMEE guide No. 14: Outcome-based education: Part 2-Planning, implementing and evaluating a competencybased curriculum. Med Teach. 1999;21(1):15-22.

3. Simpson JG, Furnace J, Crosby J, Cumming AD, Evans PA, Friedman Ben David M, et al. The Scottish doctor-learning outcomes for the medical undergraduate in Scotland: a foundation for competent and reflective practitioners. Med Teach. 2002 Mar;24(2):136-43.

4. Beneitone P. Esquetini C, González J, Marty Maletá M, Siufi G, Wagenaar $\mathrm{R}$, ed. Reflexiones y perspectivas de la educación superior en América Latina. Informe final - Proyecto Tuning América Latina 2004-2007. Bilbao: Tuning project; 2007.

5. Cieza Zevallos JA, León Rabanal CP, Huapaya Carrera JA, Miyahira Arakaki JM. Examen nacional de medicina en Perú 2008-2009: análisis y sostenibilidad de resultados. Acta Med Per. 2010 Abr-Jun;27(2):99-104.

Correspondencia: María Paola Lucía Llosa Isenrich

Dirección: Facultad de medicina, Universidad Peruana Cayetano Heredia. Av. Honorio Delgado 430, Urb. Ingenieria san Martín de Porres, Lima, Perú.

Correo electrónico:maria.llosa@upch.pe Teléfono: 999707301 